\title{
ÉPISTÉMOLOGIE
}

PAR RODOLPHE DURAND

\section{Hommage à Roland Calori}

Roland Calori a contribué par ses nombreux travaux à la fertilisation croisée des thèmes de la représentation des dirigeants comme base de leurs actions et de la conception théorique de la dynamique économique internationale.

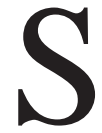
es publications (voir ci-dessous une sélection des plus récentes d'entre elles) cherchent d'une part, à insuffler la philosophie du sujet agissant dans le management. Comprendre l'acteur intimement dans ses raisons d'agir, ses raisons d'être, et sa force à devenir quelque chose ou quelqu'un d'autre par ses actes. D'autre part, elles s'efforcent de penser l'apport de la délibération et de l'échange contradictoire afin de prendre de bonnes décisions. Il nous faudrait sortir de la logique aristotélicienne ou une chose n'est que ce qu'elle est et ne peut être son contraire. Il nous faut penser l'être et les choses dialectiquement comme pouvant être ellesmêmes et s'opposer à elles-mêmes au cours du temps et pour un individu au moment de l'action. La délibération, l'écoute de l'argument opposé, la magnanimité sont des pratiques de management qu'ils fondent le critère à partir duquel on peut juger si une décision est bonne ou non. Ainsi, les travaux les plus récents de Roland, au nombre duquel ce texte ici reproduit, introduisaient au cœur de la pensée évolutionniste non pas l'utilitarisme, la recherche d'intérêts individuels mais l'ouverture au discours de l'autre, l'altruisme humaniste qui le caractérisait. Il travaillait ainsi à compléter la compréhension du discours stratégique, exprimé par les dirigeants, non plus seulement à partir de leurs cartes mentales et cognitives mais aussi de leurs valeurs intérieures, de leurs paroles en tant que messages exprimant la justice et l'injustice, le plaisir et la douleur, ou la passion et l'ennui. 
12 Revue française de gestion

\section{SÉLECTION DE PUBLICATIONS}

Calori R., Johnson G., Sarnin P., « CEOs' cognitive maps and the scope of the organization », Strategic Management Journal, vol. 15, 1994, p. 437-458.

Calori R., Dufour B., « Managing European Style », Academy of Management Executive, vol. 9, 1995, p. 61-80.

Calori R., « Philosophizing on strategic management models », Organization Studies, ${ }^{\circ}$ 19, 1998, p. 281-306.

Lubatkin M., Calori R., Very P. and Veiga J., « Managing mergers across borders: a two nation exploration of a nationally-bound administrative heritage », Organization Science, $\mathrm{n}^{\circ}$ 9, 1998, p. 670-684.

Calori R., Atamer T. et Nunes P., The Dynamics of International Competition: from practice to theory, London, Sage Publications, 2000.

Calori R., « Ordinary theorists in mixed industries », Organization Studies, n 21, 2000, p. 1031-1057.

Calori R., « Organizational development and the ontology of creative dialectical evolution », Organization, $\mathrm{n}^{\circ}$ 9, 2002, p. 127-150. 


\section{Philosophie et développement organisationnel : \\ Dialectique, agir communicationnel, délibération et dialogue \\ Roland Calori*}

Dans ce chapitre nous relions la capacité de développement d'une organisation (plus précisément sa capacité d'apprentissage) avec certaines capacités cognitives et communicatives de ses membres influents. La référence à plusieurs œuvres philosophiques permet d'identifier deux niveaux : (1) les capacités logiques et les rationalités sociologiques et (2) les pratiques de communication des individus. La « logique dialectique » est définie à partir de «Science de la Logique » de Hegel et de «l'hyperdialectique » selon Merleau-Ponty et Gurvitch. Le concept d' « agir communicationnel » est défini à partir de la « Théorie de l'Agir Communicationnel » de Habermas. Les concepts de dialogue et de délibération, définis à partir de « l'Art de la Rhétorique » d'Aristote et de la « Nouvelle Rhétorique », ont été déjà partiellement intégrés à la littérature sur l'apprentissage. Nous suggérons que chez un même individu et, par agrégation dans une organisation, les capacités logiques, les rationalités sociologiques et les capacités communicationnelles sont liées entre elles et liées aux capacités d'exploration et d'exploitation de l'organi- sation dans son ensemble (March, 1991). La théorie des organisations et le management stratégique reconnaissent la nécessité d'une coexistence des capacités d'exploitation et d'exploration; cependant la compréhension de la dynamique d'exploration (émergence, innovation, conception, création de savoir, etc.) est encore mal maîtrisée. Les conclusions formulées dans ce chapitre suggèrent que le management stratégique, par définition, est biaisé par une logique binaire et par une rationalité sociologique d'agir stratégique alors que l'exploration est fondée sur une logique dialectique et une rationalité sociologique d'agir communicationnel.

Dans les deux premières sections de ce chapitre, nous présentons deux contributions philosophiques susceptibles d'enrichir la compréhension de l'apprentissage organisationnel : les bases de la dialectique et la théorie de l'agir communicationnel. La logique constitue l'outil de base de nos raisonnements et de la compréhension de nos relations aux autres et aux objets dans l'espace et dans le temps. Par opposition à la logique binaire (logique d'identité), nous tentons d'expliquer les vertus de la logique

* Texte paru dans : Développement de l'Organisation, Nouveaux Regards, dirigé par R. Durand, Economica, 2002, p. $185-216$. 
dialectique (logique du mouvement) fondée par Hegel dans Science de la Logique $(1816,1972,1981,1989)$ et de « l'hyperdialectique » selon Merleau Ponty (1964) et Gurvitch (1962). Puis nous nous intéressons à la rationalité sociologique des membres de l'organisation. La « Théorie de l'Agir Communicationnel » développée par Jürgen Habermas $(1981,1984,1987)$ offre une différenciation claire entre l' « agir stratégique » et l' «agir communicationnel » et explique les vertus de cette dernière rationalité. La contribution d'Habermas fournit un pont entre la théorie de la conscience (philosophie) et la théorie de la communication (sociologie); en ce sens elle est au centre de notre discussion sur les capacités individuelles et organisationnelles d'exploitation et d'exploration. "L'agir communicationnel » apparaît comme le fondement du processus d'exploration.

Dans la troisième section, au sein du vaste domaine traitant du développement organisationnel, nous identifions le domaine théorique qu'il est possible d'enrichir par ces contributions philosophiques, la théorie de l'« apprentissage organisationnel », composante de la théorie de la firme fondée sur la connaissance (knowledge-based theory of the firm). Les différentes perspectives de ce courant s'accordent pour reconnaître le caractère fondamental des capacités et pratiques de communication pour l'apprentissage : la discussion ouverte et le dialogue. Une ouverture vers la rhétorique (qui constitue d'ailleurs une première fertilisation philosophique) nous permet de définir quatre formes de communication : la démonstration, l'argumentation fermée, la délibération (ou argumentation ouverte) et le dialogue.

Dans la quatrième section, nous résumons les liens entre les formes de communica- tion, les rationalités sociologiques et les formes de logique qui les sous-tendent. La découverte de ces racines enrichit la théorie de l'apprentissage organisationnel (et individuel) et la compréhension des processus de développement organisationnel.

\section{Logique dialectique et métalogique hyperdialectique}

$\mathrm{Au}$ sein de la philosophie, la logique est la discipline, fondée sur des règles formelles, qui étudie le processus de pensée quel qu'en soit le contenu. C'est l'« organon », l'instrument fondamental de la pensée et de la science et le préambule à toute philosophie (Aristote).

\subsection{Logique binaire}

La logique binaire, aussi appelée logique formelle, considère chaque énoncé comme vrai ou faux. L'ordinateur digital avec ses séries rapides binaires composées de « 1 » et de « 0 » est l'emblème de la logique formelle. La logique formelle selon Aristote repose sur une loi : « $\mathrm{A} »$ ou « non- $\mathrm{A} »$, , ceci est » ou « ceci n'est pas ». La logique binaire est fondée sur le principe d'identité, de permanence et d'exclusion du milieu (Ford et Ford, 1994). Elle n'est pas concernée par la formalisation $\mathrm{du}$ changement, toutefois, selon la logique binaire, le changement est interprété comme un remplacement : suppression de « $\mathrm{A} »$, remplacement par « B » (Ford et Backoff, 1988) et l'ambiguïté est ignorée.

Les membres d'une organisation (dont les managers) qui raisonnent suivant les règles de la logique binaire pensent aux stratégies en termes de vérités alternatives exclusives (idées et moyens d'actions); ils pensent en termes de «soit..., soit...» (ceci ou cela). Ils perçoivent le changement comme un changement douloureux de vérité, qu'il est 
préférable d'éviter tant qu'une preuve de fausseté n'a pas été démontrée (logiquement et/ou par le résultat d'actions).

Le fragment suivant de monologue par un dirigeant (probablement inspiré par Michael E. Porter) illustre cette forme de logique : « L'entreprise qui est « stuck-in-themiddle » doit faire un choix clair et net, soit elle tente de devenir le leader en coût, soit elle doit se focaliser sur un segment particulier, soit elle doit réussir à se différencier des concurrents d'une manière ou d'une autre Une fois que vous êtes « stuck-in-themiddle » ça prend du temps et beaucoup d'efforts pour s'en sortir. [...] Si vous essayez de combiner une stratégie de coût avec une stratégie de différenciation vous êtes condamnés à l'échec ».

Un tel processus de réflexion se traduit généralement par une rhétorique de démonstration de la vérité aux autres membres de l'organisation, et se fonde sur des preuves déduites et/ou démontrées par les résultats d'actions passées, c'est-à-dire sur une rationalité sociologique d' " agir stratégique » selon les termes choisis par Jürgen Habermas (1981, 1987). « L'agir stratégique » est défini par le concept de décision parmi des voies d'action alternatives, pour atteindre un but désiré par l'acteur.

\subsection{Logique dialectique}

La logique dialectique conçoit le changement comme le résultat de tensions bipolaires entre forces opposées. Pour Hegel (1816, 1972, 1981, 1989), penser comprend trois opérations distinctes : la compréhension, le raisonnement dialectique et le raisonnement spéculatif. Par la compréhension, nous définissons ou déterminons un concept. Le raisonnement dialectique est le mouvement de la pensée qui répond à la limite définie par la compréhension en allant vers le concept opposé qui se trouve au-delà de cette limite. Le raisonnement spéculatif réfléchit au mouvement complet du concept original vers son opposé et au retour vers le concept original (le négatif du négatif), établissant ainsi une perspective d'ensemble qui explique comment les deux contraires se combinent dans une unique pensée complexe (Burbidge, 1993).

Les principes de la logique dialectique hégélienne ont été appliqués pour expliquer la dynamique de la société et des organisations (par exemple Benson, 1977). La traduction de ce processus de raisonnement dans les théories du changement social a produit les idées suivantes : le changement est généré par la tension entre des forces opposées, une pression extrême (en quantité) d'une force sociale sur son opposant conduit à un changement en qualité, une révolution dans laquelle la forme dominante précédente est niée. La pensée dialectique a été (et est encore) souvent critiquée pour la fermeture que semble imposer la synthèse au mouvement thèse-antithèse et pour l'impression de progression inéluctable et idéaliste vers « l'idée absolue ». Nous soutenons (avec d'autres philosophes lecteurs de Hegel) que ces critiques ne sont pas fondées. Hegel n'a jamais écrit que le concept original et son négatif sont perdus dans une synthèse : «Le négatif du négatif consiste à unifier une paire donnée de concepts contraires qui s'impliquent mutuellement, dans un sens en les préservant et dans un sens en les abolissant, éliminant ainsi leur caractère contradictoire, tout en étant proche des deux en termes de contenu » (Forster, 1993 p. 148). En outre, la lecture de Science de la Logique, ouvrage dans lequel Hegel explique dans le détail la logique dialectique, montre que l'idée abso- 
lue n'est autre et toujours que l'idée de mouvement continuel entre l' «idée théorique » et l'« idée pratique » (donc certainement pas une fermeture). La dialectique de «l'être » révèle les principes de cette logique, et deux concepts, devenir et relier, sur lesquels une théorie du renouveau organisationnel peut être construite.

\subsection{Dialectique de l'être}

La dialectique de l'être : « Être-NéantDevenir » est la composante élémentaire de toutes les pensées, elle définit les termes de la logique dialectique hégélienne. Elle est le point de départ de nombreux questionnements ontologiques et logiques. Elle comprend trois étapes : la compréhension de «l'être », de « l'être-là » et de « l'être poursoi », résumées dans le schéma 1.

\section{L'être}

Quand nous pensons à « l'être » comme idée pure, hors de tout contexte, nous réalisons que nous commençons à penser "néant ", car sans détermination de l'être, il n'y a rien à penser. Le « néant » aussi est indéterminé, comme «l'être », mais en même temps c'est aussi quelque chose à quoi nous pensons, et penser au «néant » nous renvoie à «l'être». " L'être pur et le néant pur sont la même chose. Ce qui est la vérité, ce n'est ni l'être ni le néant, mais le fait que l'être non point passe mais est passé en néant, et le néant en être. Pourtant la vérité, tout aussi bien, n'est

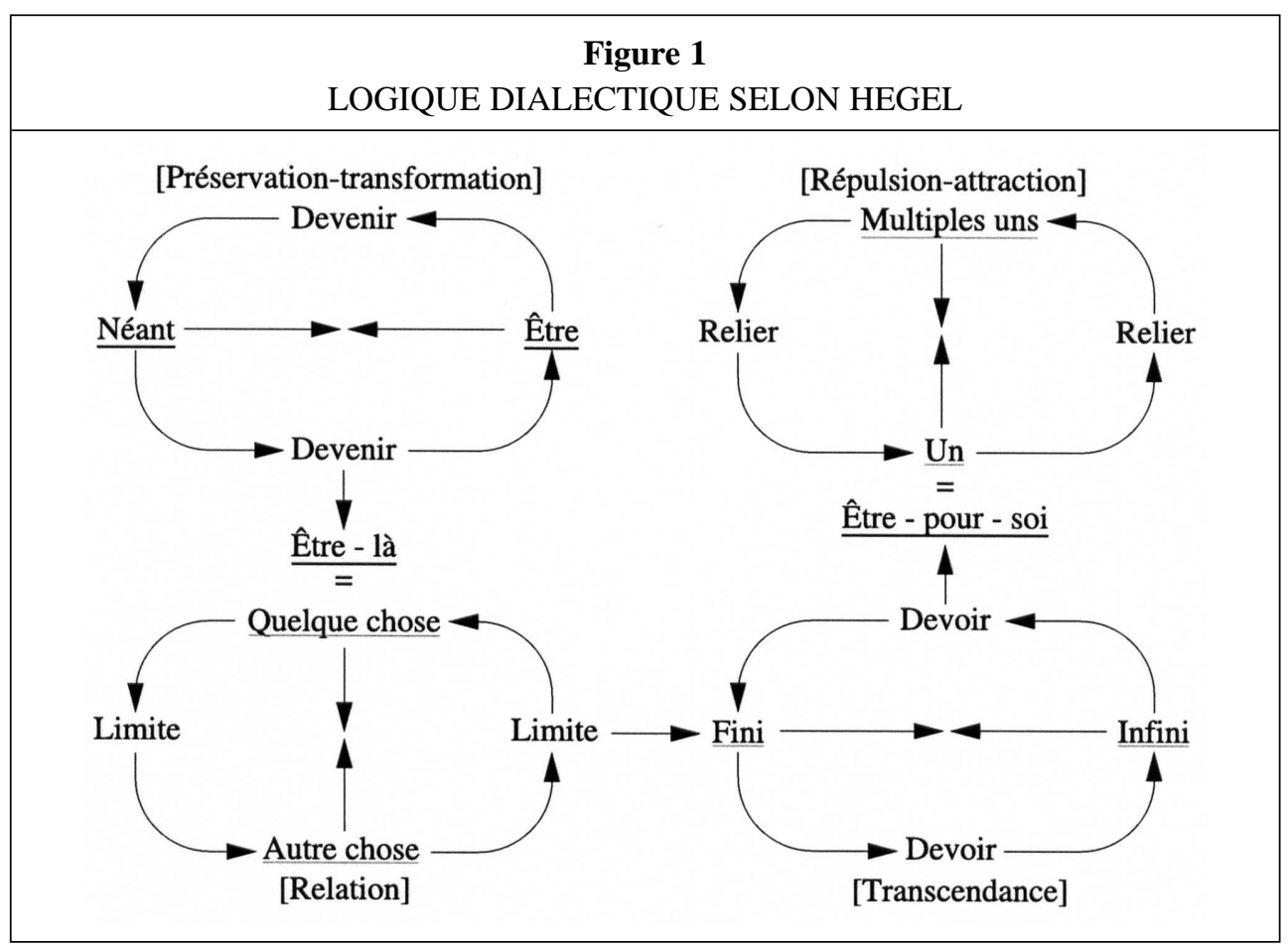


pas leur état-de-non-différenciation, mais le fait qu'ils sont absolument différents, et que pourtant, tout aussi immédiatement, chacun disparaît dans son contraire. Leur vérité est donc ce mouvement du disparaître immédiat de l'un dans l'autre; le devenir; un mouvement où les deux sont différents, mais par le truchement d'une différence qui s'est dissoute tout aussi immédiatement » (Hegel, 1816,1972 , p. 23-24). Devenir existe en pensée comme un moment dans la transition, un « être-là » (dasein en allemand, determinate being en anglais).

\section{L'être-là}

«L'être-là » est l'unité dans laquelle l'être et le néant (contraires) sont préservés, à un certain moment en un certain lieu, le point de départ d'un nouveau mouvement de la pensée. À un certain moment en un certain lieu, l'être-là peut être qualifié et devient «quelque chose ». Le négatif de « quelque chose » est une « autre chose ». Une « autre chose » est aussi immédiatement «quelque chose ». Par sa nature même, " quelque chose » est lié à « autre chose ». La « limite » est le point qui sépare les deux, qui les distingue et qui permet de les définir tous les deux, les «quelque chose » sont « finis ». Dans le « devoir être » (ought) commence la transcendance de la finitude, le « devoir-être » rend possible en pensée le progrès impossible vers l'infinité qui est un pur idéal. L'être-là qui dépasse la limite devient «être-pour-soi », le négatif se rapportant à soi, « l'être déterminé absolu ».

\section{L'être-pour-soi}

«L'être pour-soi » (fürsichein en allemand, being-for-self en anglais) est d'abord et immédiatement un être-pour-soi : un «Un ». La relation négative de l'un avec soi-même est la répulsion qui le fait sortir de soi-même. Ce sortir-de-soi conduit à de «multiples uns », d'abord repoussés et niés. La répulsion définit ainsi la relation entre les «multiples uns ». Les multiples uns se préservent par leur répulsion réciproque. Puis la négation de la répulsion permet de retrouver l'être-pour-soi. « [...] La répulsion est elle-même relation : l'un qui exclut les uns se relie à eux, c'est-à-dire à luimême. Donc la relation négative des uns entre eux n'est qu'un mouvement ensemble avec soi. [...] Ce mouvement des multiples uns dans un seul un est attraction [...] (Hegel, 1816, 1989, p. 171-172).

La répulsion de la répulsion devient attraction, s'il n'y avait pas de multiples uns il n'y aurait pas matière à attraction. Si l'on concevait l'attraction comme totalement accomplie, les multiples uns fusionnés dans un seul un, il n'y aurait plus qu'un un inerte. De même si l'on concevait la répulsion comme totalement accomplie, les multiples uns dispersés et non reliés, il n'y aurait plus qu'un vide inerte. L'unité parfaite engendre l'inertie, la division parfaite engendre le vide, la pluralité engendre le mouvement. $\mathrm{La}$ 《 relation » (relier) est une condition du mouvement, d'abord par la reconnaissance des limites qui définissent l'être à un moment et en un lieu donné par rapport à l'autre et à soi-même, et par les tensions, répulsionattraction, qui caractérisent l'être-pour-soi et le relient aux autres et à soi-même. Le « devenir » exprime le mouvement de l'être par le devoir qui le pousse à transcender ses limites et par les tensions, préservation-transformation, qui le poussent à la relation avec les autres et avec soi-même. Les concepts de devenir et relier définissent les mouvements au cœur de la logique dialectique, ils sont liés entre eux par la chaîne d'idées décrite en 
schéma 1, suivant les trois étapes de la détermination de l'être.

De cette analyse il ressort également que les nombreuses critiques habituelles faites à la dialectique Hégélienne sont injustes : la synthèse n'abolit pas les termes d'origine et le conflit (la répulsion) est inséparable de l'attraction réciproque. Adorno (1984) a clairement confirmé cette conclusion : « la notion de synthèse n'est pas un moment de fermeture ou d'oblitération des pensées précédentes, au contraire, elle représente une opportunité infinie d'ouverture, car tout ce qui est nouveau préserve les vieilles contradictions dans la mesure où elles sont médiatisées dans le nouveau » (Adorno, 1984, p. 38). Aussi étonnant que cela puisse paraître, comme le notent Modell (1996) et Carr (2001): Hegel a été le premier «psychologue de l'intersubjectivité et de la relation ». Pour lui, la réalité du soi, la conscience de soi, requiert la reconnaissance par une autre conscience de soi (une relation).

Les membres d'une organisation qui pensent selon les principes de la logique dialectique sont ouverts aux oppositions, aux contradictions et aux argumentations avec les autres. La référence au dialogue est encore plus nette chez d'autres philosophes concernés par le mouvement, et qui proposent des dialectiques sans synthèse appelée « hyperdialectiques » (Gurvitch, 1962; Merleau-Ponty, 1964).

\subsection{Métalogique hyperdialectique}

Plusieurs modes de raisonnement « hyperdialectiques, » permettent de comprendre les phénomènes sociaux complexes, il ne s'agit plus de logique au sens strict mais plutôt de «métalogiques » (telles que définies par Morin, 1991, p. 194) qui rendent compte des multiples relations possibles entre concepts opposés. Gurvitch (1962) identifie cinq procédés de raisonnement hyperdialectique : la polarisation, l'ambiguïté, la complémentarité, l'implication mutuelle et la réciprocité de perspective.

La polarisation dialectique correspond à des antinomies et à des tensions entre opposés telles que les conçoit la logique dialectique Hégélienne; la résolution des tensions s'exprime alors dans le mouvement entre les termes opposés. L'ambiguïté dialectique est un processus de raisonnement qui reconnaît l'ambiguïté de concepts opposés, voire leur ambivalence dans une harmonie des contraires. Par exemple, les relations entre soi-même et les autres peuvent être ambivalentes, à la fois composées d'attrait et de répulsion. Merleau-Ponty (1964) utilise la métaphore du « chiasme »- point de contact entre deux chromosomes lorsqu'ils échangent leurs segments lors de la formation de cellules reproductrices - pour représenter l'ambivalence dialectique qui différencie et unifie les contraires dans un mouvement continuel. La complémentarité dialectique est un procédé de raisonnement qui dévoile le caractère complémentaire de deux termes (à l'origine considérés comme contraires) dans un même tout. Il peut s'agir d'alternatives qui se révèlent complémentaires, par exemple les perspectives économique et sociale. Il peut s'agir de concepts a priori opposés qui se compensent et se régénèrent mutuellement, par exemple l'organisation et la spontanéité. Il peut s'agir de doubles mouvements de termes opposés parfois dans des directions opposées et parfois dans la même direction, par exemple les compensations entre les forces au centre et les forces à la périphérie d'une organisation. L'implication dialectique mutuelle révèle, dans des termes à 
première vue hétérogènes ou contraires, des composantes qui se recoupent, se contiennent et s'interprètent à un certain degré; par exemple la vie psychique et la vie sociale s'interprètent suggérant ainsi une approche psychosociale pour comprendre les comportements humains. La mise en réciprocité de perspectives fait ressortir dans des éléments n'admettant ni identification, ni séparation, leur immanence réciproque devenue si intense qu'elle conduit à un parallélisme ou à une quasi symétrie entre leurs manifestations; par exemple les tensions au niveau collectif entre groupes sociaux (producteurs et consommateurs ou autres) se retrouvent au niveau individuel où chacun est appelé à jouer des rôles sociaux opposés. La compréhension de telles symétries aide à réconcilier certaines oppositions.

Comme nous le verrons plus loin tous ces procédés de raisonnement hyperdialectiques favorisent l'attention portée à la différence et à la variété. La compréhension d'ambivalences, de complémentarités, d'implications mutuelles et de réciprocités rend possibles les dialogues dans lesquels les uns et les autres suspendent leurs certitudes, le temps de considérer des termes opposés et de les combiner éventuellement dans une pensée plus complexe.

Les fragments suivant d'un monologue par le directeur général de Salomon (leader mondial de l'équipement de sports d'hiver) illustrent les traces de logique dialectique et de raisonnement hyperdialectique qu'il est possible de trouver dans le discours d'un praticien :

"Le succès a ses dangers, les gens commencent à croire que c'est gagné pour toujours. Leur excitation réduit leur vigilance. Vous savez, le danger des graphes et des courbes de ventes qui montent, qui mon- tent... On ne réalise pas qu'elles pourraient se stabiliser ou redescendre si on ne fait rien Pendant la période 1983-1987 beaucoup d'entre nous étaient euphoriques... Mais quelques-uns n'étaient pas totalement satisfaits. Par exemple, nous sommes fiers de notre style « commando », on a une centaine de commandos... et il y en a de plus en plus au fur et à mesure que l'on grossit, ce n'est pas facile de les faire travailler ensemble quand c'est nécessaire de former un tout. [...] Aussi vous devez acquérir de nouvelles qualités tout en ne perdant pas vos qualités d'origine... ce n'est pas facile de concilier le contrôle de gestion et les initiatives! [...] Comme vous le savez à cette époque nous nous sommes diversifiés dans les équipements de golf et nous avons investi des dizaines de millions pour développer un nouveau ski. [...] Il y a eu des débats sans fin au sujet de ces diversifications : est-ce qu'on doit y aller? Est-ce qu'on doit ne pas y aller? Il y avait de bons arguments de chaque côté Finalement on l'a fait, mais d'après moi on a perdu du temps, on aurait pu gagner un an [...]. À cette époque-là j'ai appris plusieurs choses [...] quand vous essayez de changer, - il y a toujours des désaccords. Vous n'êtes pas d'accord avec vous-même et vous n'êtes pas d'accord avec les autres, il y a des tensions, de l'anxiété... Il vous faut apprendre à vivre ensemble, mais ça doit être clair et sincère. On a besoin de fortes personnalités, mais les gens doivent aussi être capables de communiquer entre eux et de trouver un accord... sans émasculer leurs idées [...]. Vous savez les directeurs... j' aimerais bien qu'ils travaillent en équipe, mais ce n'est pas évident, c'est quand même des gens qui ont des curriculums différents, tous des tronches, si vous me permettez, ils ne sont 
pas arrivés là en étant falots... Tous des gros calibres. D'abord ils doivent comprendre qu'en travaillant ensemble ils seront plus intelligents ».

L'analyse de contenu de discours (sincères) de membres d'une organisation permet d'identifier les concepts-clés et les relations entre concepts qui forment la structure de raisonnement de l'individu sur un phénomène donné (pour un exemple, voir Calori, 2002). Il est donc possible de caractériser le type de logique d'une personne : binaire, dialectique ou hyperdialectique.

\section{Habermas : argumentation et agir communicationnel}

La théorie de l' «Agir Communicationnel » (ou « action communicationnelle ») développée par Jürgen Habermas (1981, 1984, 1987) crée un pont entre le niveau logique et les formes pratiques de communication (rhétorique et dialogue). Dans l'« Agir Communicationnel », Habermas offre une philosophie qui coopère avec la sociologie, une théorie de la société basée, sur l'action orientée vers l'intersubjectivité, actions raisonnables sujettes à la critique.

\subsection{Personnes « rationnelles» et « responsables »}

Habermas commence par une discussion sur le comportement rationnel. Il considère comme une « personne rationnelle » quelqu'un qui est responsable. Pour être responsable, il ne suffit pas qu'une personne réussisse dans ses interventions dirigées vers un but (le succès de telles interventions requiert seulement qu'elle soit capable de choisir entre des options alternatives et de contrôler quelques conditions de l'environnement). Seul/e peut être considéré/e comme responsable celui/celle qui réussit à progresser vers et à atteindre la compréhension réciproque (Verstandigung, en allemand).

Dans l'agir communicationnel, comme membre d'une communauté de communication, une personne responsable est capable d'orienter ses actions selon « des prétentions à la validité intersubjectivement reconnues » (1987, p. 31). Habermas considère quelqu'un comme rationnel s'il/elle est capable d'utiliser trois types de prétentions à la validité : (1) il/elle porte une affirmation et la fonde face à un critique en indiquant les évidences (logique et/ou efficience) qui correspondent à cette affirmation; (2) il/elle suit une norme existante et peut justifier son action face à un critique en expliquant la légitimité de ce comportement (« action régulée par des normes »); et (3) il/elle exprime un désir sincère, un sentiment sincère, révèle une expérience qui est reconnue par le critique, en tire des conséquences pratiques et se comporte en conséquence par la suite (« présentations de soi expressives »). Dans les deux dernières formes de prétentions à la validité on serait tenté d'utiliser le qualificatif « raisonnable » plutôt que « rationnel », Habermas préfère adopter un qualificatif commun pour les trois types de critères de validité, au même niveau. Dans la première forme de prétention à la validité le sujet se réfère au «monde objectif », dans la seconde le sujet se réfère au « monde social », et dans la troisième au « monde subjectif». Dans ces trois mondes une personne « responsable», donc « rationnelle », peut expliquer son comportement par plusieurs prétentions à la validité : vérité logique/efficience, normes sociales, et/ou expériences subjectives sincères.

\subsection{Argumentation}

Sur la base des trois types de prétentions à la validité, Habermas construit une théorie 
de l'argumentation. Il s'appuie sur Toulmin et al. (1979) pour définir un processus d'argumentation rationnel :

«Quiconque participe à une argumentation démontre sa rationalité ou son manque de rationalité par la manière dont il traite les raisons qu'on lui présente pour ou contre une prétention, et par la manière dont il y répond. S'il est ouvert à l'argumentation, ou bien il reconnaît la force de ces raisons, ou bien il cherche à y répliquer : de toute façon il les traite d'une manière « rationnelle ». S'il est « sourd à l' argumentation », il peut en revanche soit vouloir ignorer les raisons qui contredisent sa prétention, soit y répondre par des affirmations dogmatiques, et de toute façon il ne traite pas ces proMêmes rationnellement » (Toulmin, Reeke et Yanik, 1979, p. 34).

Les personnes qui se comportent rationnellement sont aussi prêtes à s'exposer aux critiques, et, si nécessaire à participer correctement à une argumentation : «En raison de leur caractère critiquable, les expressions rationnelles sont également susceptibles d'amélioration : nous pouvons corriger des tentatives ratées, si nous réussissons à identifier les fautes qui nous ont échappé. Le concept de fondation rationnelle est étroitement uni à celui d'apprentissage » (Habermas, 1981, 1987, p. 35). De plus, n'importe quel interprète qui se trouve en difficulté dans un processus d'intercompréhension devrait réfléchir aux problèmes communicationnels : [...] Face à la persistance des difficultés d'intercompréhension, il n'a pas d'autre recours que de prendre comme objet de la communication les moyens de l'intercompréhension (Verständigung) euxmêmes. Nous nommons rationnelle une personne qui manifeste une disposition à l'intercompréhension et réagit aux pertur- bations de la communication en réfléchissant sur les règles langagières » (Habermas, 1981, 1987, p. 38). Ici Habermas souligne l'importance de ce qui est parfois appelé « metacommunication », une communication au deuxième niveau, sur le processus de communication que les individus sont en train de vivre.

De ce qui précède, il apparaît que la logique de l'argumentation ne se fonde pas uniquement sur des déductions (comme le fait la logique formelle binaire), elle se fonde aussi sur des raisonnements non-déductifs entre les actes de parole qui composent les arguments. Il apparaît aussi qu'une personne rationnelle ne doit accepter aucune asymétrie ou aucune domination a priori dans la communication (surtout si l'asymétrie provient d'une hiérarchie établie) : « [...] Ce qui aujourd'hui comme hier me semble juste, c'est l'intention de reconstruire les conditions de la relation symétrique, conditions que tout locuteur compétent est obligé de présupposer comme étant tendanciellement remplies, pour autant qu'il pense en général entrer dans une argumentation. Ceux qui prennent part à l'argumentation sont obligés de présupposer généralement que la structure de leur communication, dont les traits caractéristiques relèvent d'une description purement formelle, exclut toute contrainte (qu'elle provienne de l'extérieur ou du procès d'intercompréhension lui-même) - toute contrainte hormis celle de l'argument meilleur (ce qui veut dire qu'elle écarte également tous les motifs hormis celui de la recherche coopérative de la vérité). Sous cet aspect, l'argumentation peut être conçue comme une poursuite par des moyens réflexifs de l'activité orientée vers l'inter-compréhension » (Habermas, 1981, 1987, p. 41). 
Finalement, en référence aux autres niveaux de notre réflexion et à la rhétorique selon Aristote, Habermas considère que la logique binaire (formelle) traite des «produits » de l'argumentation, la logique dialectique traite des «procédures » d'argumentation, et la rhétorique traite des «processus réels » d'argumentation. La théorie de l'agir communicationnel suggère que les processus réels d'argumentation sont influencés par la procédure (logique dialectique), et que le «produit » de ces processus peut être examiné selon les critères de la logique formelle binaire. La théorie de l'agir communicationnel fournit l'explication du passage de la procédure au processus réel via la rationalité sociologique adoptée par les acteurs.

\subsection{Décentration et développement}

En accord avec Hegel (le concept de relation, la répulsion-attraction entre l'un et les autres), et en accord avec le concept d'apprentissage selon Piaget (1972), Habermas considère que chaque interaction entre sujets individuels les modifie mutuellement. Le développement cognitif « signifie en général la décentration d'une compréhension egocentrée du monde » (Habermas, 1981, 1987 p. 85, souligné par l'auteur). Chaque action orientée vers l'atteinte d'une compréhension mutuelle fait partie d'un processus coopératif d'interprétation visant des définitions de situations reconnues intersubjectivement : «La décentration de la compréhension du monde s'est révélée comme la dimension la plus importante de l'évolution des images du monde » (1981, 1987, p. 91). L'intersubjectivité combine nécessairement les mondes « objectif», « social » et « subjectit» qui ne sont mêlés que dans le «monde vécu », ainsi on ne peut atteindre la compréhension mutuelle que dans le «monde vécu » (Lebenswelt en allemand, lifeword en anglais).

En résumé, Habermas identifie les propriétés formelles d'une société (ou d'une organisation) qui a le potentiel d'apprendre et de se développer (1981, 1987, p. 87-88).

(1) Elle doit reconnaître les mondes objectifs, social et subjectif et « autoriser des prétentions différenciées à la validité, vérité propositionnelle, justesse normative, véracité subjective ».

(2) Elle doit « autoriser une relation réflexive à elle-même, être dépouillée de son dogmatisme de façon à permettre que les interprétations alimentées par la tradition soit fondamentalement mises en question et soumises à une révision critique $[\ldots] \gg$.

(3) Elle doit permettre « des argumentations spécialisées » pour des activités spécialisées : science, droit, art, administration, qui forment des sous-systèmes culturels et professionnels.

(4) Elle doit permettre que « l'activité orientée vers le succès puisse être affranchie des impératifs d'une entente toujours à renouveler dans la communication, et qu'elle puisse être détachée du moins partiellement de l'activité orientée vers l'entente intersubjective. C'est ainsi que devient possible, en vue d'objectifs généralisés, l'institutionnalisation sociale de l'activité rationnelle par rapport à une fin; il peut s'agir par exemple de la constitution de sous-systèmes régis par l'argent et le pouvoir aux fins de l'économie rationnelle et de l'administration rationnelle » (1987, p. 88).

Dans l'énoncé de cette quatrième propriété, dans le cas particulier du management des organisations (régies par l'argent, le pouvoir, l'économie et l'administration rationnelles), Habermas reconnaît donc à la fois 
« l'agir stratégique » et « l'agir communicationnel ». Cependant seul l'agir communicationnel possède les quatre propriétés qui permettent l'apprentissage.

\subsection{Agir communicationnel}

Suivant les prétentions à la validité quelles mobilisent, Habermas identifie quatre rationalités sociologiques : l'agir communicationnel, l'agir stratégique, l'agir régulé par des normes, et l'agir dramaturgique. L'agir dramaturgique est un système de rationalité dans lequel chaque agent communique aux autres ses propres intentions, pensées, attitudes, désirs, sentiments, exprime sincèrement ses propres expériences et se réfère uniquement au monde subjectif. L'agir régulé par des normes est un système de rationalité dans lequel les membres d'un groupe social orientent leurs actions dans le sens de valeurs communes, se conforment ainsi à une norme sociale de comportement, et se réfèrent au monde objectif et au monde social.

Dans le contexte qui nous intéresse, le management et le développement d'une organisation, la comparaison entre l'agir stratégique et l'agir communicationnel est la plus instructive.

«L'agir stratégique » est un modèle d'action téléologique dans lequel chaque agent calcule les moyens et les fins pour parvenir au succès (l'atteinte de ses buts), en anticipant les décisions des autres acteurs, eux aussi orientés vers leurs propres buts. Dans l'agir stratégique les agents se réfèrent uniquement au monde objectif et utilisent deux types de prétentions à la validité dans leur argumenta- tion : la logique propositionnelle et/ou l'efficience (évidence empirique de succès).

« L'agir communicationnel » est la seule rationalité sociologique qui se réfère aux trois mondes : objectif, social et subjectif ${ }^{1}$. Dans ce type d'interaction les participants reconnaissent intersubjectivement les prétentions à la validité que chacun mobilise. Dans le but coopératif d'atteindre une compréhension réciproque les acteurs utilisent trois types de prétentions à la validité : l'énoncé est «vrai », l'acte de parole est « juste » en référence au contexte normatif existant, et l'intention de l'interlocuteur est « sincère » (exprimée comme elle est pensée). Chaque acteur s'engage alors à essayer de comprendre les mondes objectif, social et subjectif de l'autre autant que le sien. Ce processus d'interprétation mutuelle se réalise par le langage et par des actions coordonnées dans le monde vécu.

En comparaison, dans le modèle d'agir stratégique chaque acteur est orienté vers son propre succès et ne se conduit de façon coopérative que dans le cas où la coopération sert son propre calcul égocentrique d'utilité Le modèle téléologique d'agir stratégique « recourt au langage comme à un médium parmi plusieurs autres, à travers lequel les locuteurs $[\ldots]$ influent les uns sur les autres pour inciter le partenaire à former ou concevoir les opinions ou intentions souhaitées au regard de leur propre intérêt » (Habermas, 1981, 1987, p. 111). Dans cet objectif, l'acteur engagé dans l'agir stratégique accepte l'asymétrie dans la communication, la domination exercée par $\mathrm{n}$ acteur

1. «Enfin, le concept de l'agir communicationnel concerne l'interaction d'au moins deux sujets capables de parler et d'agir qui engagent une relation interpersonnelle (que ce soit par des moyens verbaux ou extra-verbaux). Les acteurs recherchent une entente (Verständigung) sur une situation d'action, afin de coordonner consensuellement (einvernehmlich) leurs plans d'action et de là même leurs actions. Le concept central d'interprétation intéresse au premier chef la négociation de définitions de situations, susceptibles de consensus. Dans ce modèle d'action, le langage occupe, comme nous le verrons, une place prééminente »(Haberrnas, 1981, 1987, p. 102). 


\begin{tabular}{|c|c|c|}
\hline \multicolumn{3}{|c|}{$\begin{array}{c}\text { Tableau } 1 \\
\text { COMPARAISON ENTRE L' «AGIR COMMUNICATIONNEL » } \\
\text { ET L'«AGIR STRATÉGIQUE »SELON HABEMAS }\end{array}$} \\
\hline & Agir stratégique & Agir communicationnel \\
\hline $\begin{array}{l}\text { Comportement } \\
\text { rationnel }\end{array}$ & $\begin{array}{l}\text { Une personne rationnelle est une } \\
\text { personne qui lorsqu'elle avance une } \\
\text { assertion qui est critiquée, est capable } \\
\text { de la fonder sur l'évidence (vrai). Les } \\
\text { prétentions à la validité se réfèrent au } \\
\text { " monde objectif »: logique de la } \\
\text { proposition et/ou efficacité empirique. }\end{array}$ & $\begin{array}{l}\text { Une personne « rationnelle » (res- } \\
\text { ponsable) est une personne qui réussit } \\
\text { dans ses processus visant la compré- } \\
\text { hension mutuelle, oriente ses actions } \\
\text { en fonction de critères de validité } \\
\text { reconnus «intersubjectivement ». } \\
\text { Les prétentions à la validité se réfèrent } \\
\text { au « monde objectif » (vrai), au } \\
\text { «monde social» (normes justes) et au } \\
\text { " monde subjectif » (expériences } \\
\text { subjectives sincères). }\end{array}$ \\
\hline Argumentation & $\begin{array}{l}\text { L'objet de la communication est } \\
\text { d'atteindre un but et de convaincre } \\
\text { l'autre. } \\
\text { Accepte l'asymétrie entre les } \\
\text { personnes engagées, dont l'asymétrie } \\
\text { fondée sur une hiérarchie établie. }\end{array}$ & $\begin{array}{l}\text { L'objet de la communication est } \\
\text { d'atteindre une compréhension } \\
\text { mutuelle. } \\
\text { N'accepte pas la domination d'une } \\
\text { personne dans la communication } \\
\text { (particulièrement quand l'asymétrie } \\
\text { est fondée sur une hiérarchie établie). } \\
\text { Recherche et pratique de la symétrie. }\end{array}$ \\
\hline $\begin{array}{l}\text { Position } \\
\text { du sujet }\end{array}$ & $\begin{array}{l}\text { Compréhension égocentrique du } \\
\text { monde. }\end{array}$ & Décentration du sujet. \\
\hline Modèle & $\begin{array}{l}\text { Téléologique, chaque agent calcule les } \\
\text { moyens et les fins pour atteindre le } \\
\text { succès (ses propres buts), tente } \\
\text { d'anticiper les décisions des autres } \\
\text { acteurs orientés vers leurs propres } \\
\text { buts, et essaie d'influencer l'autre (cf. } \\
\text { asymétrie). }\end{array}$ & $\begin{array}{l}\text { Les acteurs cherchent à coordonner } \\
\text { leurs actions par le moyen de l'accord } \\
\text { mutuel, qui commence par la négocia- } \\
\text { tion des interprétations mutuelles (cf. } \\
\text { symétrie). }\end{array}$ \\
\hline $\begin{array}{l}\text { Forme de } \\
\text { communication }\end{array}$ & Démonstration, discussion fermée. & Dialogue, discussion ouverte. \\
\hline
\end{tabular}

sur un autre, éventuellement fondée sur une hiérarchie formelle. Au contraire, dans l'agir communicationnel les acteurs s'efforcent de créer et de préserver une condition fondamentale de symétrie entre les interlocuteurs.
Le tableau 1 résume la comparaison entre les eux rationalités sociologiques.

Il est possible d'identifier le mode dominant de rationalité sociologique d'un individu (et de l'ensemble des individus dans un groupe 
et/ou une organisation) à partir de l'analyse de conversations : les types de prétentions à la validité qu'il/elle utilise et qu'il/elle accepte, sa sincérité, le poids de ses intérêts personnels, le respect ou non du principe de symétrie (Samra-Fredericks, 2001).

Les relations entre le niveau logique (section I) et le niveau de la rationalité sociologique peuvent être déduites des descriptions respectives de ces processus de raisonnement et d'argumentation. Un individu adoptant un mode de logique binaire, qui distingue nettement le vrai du faux, aura tendance à adopter un mode d'agir stratégique dans lequel il déploiera tous ses arguments pour convaincre l'autre et atteindre son but. Un individu adoptant un mode de logique dialectique, qui accepte la contradiction comme source de mouvement et de progrès, aura tendance à se décentrer, accepter la critique, s'ouvrir à des arguments de diverses natures, tenter de comprendre l'autre subjectivement, en bref à adopter un mode d'agir communicationnel. À l'extrême, un individu adoptant un mode de logique hyperdialectique dans lequel les opposés coexistent en tension ou au repos, aura tendance à adopter le mode le plus pur d'agir communicationnel, qui vise essentiellement la compréhension mutuelle intersubjective. Puisque nous nous intéressons à des organisations à vocation économique régies par l'argent (et pas seulement sociales), comme le précise Habermas dans la quatrième propriété d'une société capable d'apprendre et de se développer : l'individu et l'organisation devraient être capables de faire coexister, selon les moments, une rationalité d'agir stratégique et une rationalité d'agir communicationnel. Comme nous allons le voir dans la section suivante, les deux modèles - agir straté- gique $v s$ agir communicationnel - se traduisent par des pratiques et des formes de communication particulières évoquées dans la littérature en management stratégique et développement organisationnel. L'agir communicationnel défini par Habermas, découvre les racines conceptuelles et cognitives des pratiques de dialogue et de délibération (argumentation ouverte), selon un principe de symétrie et de justice. L'agir stratégique défini par Habermas, découvre les racines conceptuelles et cognitives des pratiques d'argumentation fermée et de démonstration. Enfin, la coexistence d'agir stratégique et d'agir communicationnel pourrait être une condition de base de la coexistence de capacités d'exploitation et d'exploration au niveau de l'organisation.

\section{Développement organisationnel, apprentissage et formes pratiques de communication}

Le terme « développement organisationnel » est ici employé dans un sens générique et nous adoptons une définition schumpétérienne (Schumpeter, 1934, 1989). Le développement est accompagné par la croissance mais la seule croissance quantitative ne suffit pas à définir le développement, qui a trois caractéristiques principales : il provient de l'intérieur du système (l'organisation) et pas seulement comme une adaptation à des changements de l'environnement, il se produit de façon discontinue plus souvent que de façon progressive et il apporte des changements qualitatifs qui modifient les anciens équilibres et créent des conditions radicalement nouvelles. Le vaste champ d'étude du développement organisationnel se compose de plusieurs grands courants : (l) le courant «Organizational Development » (plus 
connu sous son acronyme, «OD», avec parmi ses fondateurs Kurt Lewin et le Tavistock Institute), (2) le courant « nouvelles formes organisationnelles » orchestré par Arie Lewin et représenté dans la revue Organization Science (voir les numéros spéciaux de cette revue sur « les processus de communication virtuels et en réseaux »; « les applications de la théorie de la complexité » et « la co-évolution » en 1999), (3) le courant politique, (4) le courant symbolique et (5) le courant de «l'apprentissage organisationnel $\gg$. Nous nous intéressons ici au courant de l'apprentissage organisationnel car il s'inscrit dans une perspective socio-cognitive de l'organisation (raisonnements et arguments logiques et sociologiques), et il a déjà intégré de façon marginale une perspective dialectique.

\subsection{Dialectique, développement et apprentissage : de l'individu à l'organisation}

Le raisonnement dialectique est le moteur de la formation des stratégies à la fois délibérées et émergentes (Mintzberg et Waters, 1985), globales et locales (Prahalad et Doz, 1987). Dans ces cadres de référence, l'orientation d'une organisation résulte de négociations et discussions entre groupes au point de vue opposés. Les processus délibérés orchestrés par le sommet de la hiérarchie sont effectivement composés de délibérations pour orienter l'action, et les idées émergeant de l'action n'importent où dans l'entreprise font aussi l'objet de délibérations qui peuvent conduire à les légitimer, généralisant ainsi le processus d'apprentissage. La perspective dialectique souligne le besoin, à la fois, d'un certain niveau de consensus et d'un certain niveau de dissension, d'un certain niveau de satisfaction et d'un certain niveau d'insatisfaction sources de l'innovation (Hedberg, Nyström et Starbuck, 1976; Evans et Doz, 1989). En général cependant, dans le domaine du management stratégique, les modèles fondés sur une dynamique dialectique sont relativement rares : les tensions entre les forces d'intégration globale et les forces d'adaptation locales (Prahalad et Doz, 1987), les tensions créatrices entre les opposés et le dépassement des dilemmes (Hampden-Turner, 1990), et la dynamique d'exploration-exploitation (March, 1991) sont les plus connus. Si l'on se réfère à la classification proposée par Van de Ven et Poole (1995), la perspective téléologique et la perspective évolutionniste dominent le management stratégique et les théories du développement organisationnel. Les théories téléologiques reconnaissent le rôle crucial des architectes de la stratégie, de «l'enactement » volontariste et du consensus. Elles expriment des tensions entre un futur désiré et la réalité présente perçue par les architectes de la stratégie. Les théories évolutionnistes reconnaissent le déterminisme environnemental dans un processus de variation ( «aveugle ») - sélection - rétention (Hannan et Freeman, 1977; voir le chapitre 1 de cet ouvrage). Elles expriment des tensions entre les forces de l'environnement et les forces organisationnelles. Ces théories négligent cependant les tensions créatrices entre les membres de l'organisation.

Dans la plupart des entreprises, la probabilité d'un consensus spontané entre les acteurs sur une question nouvelle est relativement faible (à moins que l'entreprise ait déjà parfaitement imprimé son code dans les cerveaux de tous ses membres). Alors le débat dialectique devient une source d'apprentissage de nouvelles idées et de coordination d'actions nouvelles (irréalisables 
selon les routines déjà instituées). Dans les cas extrêmes, sans contradiction (sans dissension) l'entreprise court le risque d'Icare Miller, 1990) : une dégénérescence progressive résultant d'une pensée collective homogène, inspirée des recettes des succès passés que l'on ne remet pas en question. Dans la perspective dialectique du développement organisationnel, les conflits ne se terminent pas par la destruction d'une des forces en opposition, il s'agit plutôt de tensions internes à l'origine de tout mouvement, tels que les conçoivent Hegel et Merleau-Ponty. La plupart des auteurs qui défendent les vertus de la contradiction, du dialogue et de la coordination traitent de l'apprentissage individuel et organisationnel dans le cadre de la « théorie de la firme fondée sur la connaissance » (« knowledge-based theory of the firin » en anglais). La théorie de la firme fondée sur la connaissance (Grant, 1996a) explique la performance organisationnelle (les rentes, la croissance et le développement sur longue période) par la création et l'intégration de compétences distinctives (« distinctive knowledge »). Cette définition conduit à analyser une capacité («capability ») organisationnelle particulière : la capacité spécifique de l'organisation à intégrer des connaissances individuelles locales et distinctives (Grant, 1996a). Selon Grant certaines capacités organisationnelles d'ensemble méritent notre attention car elles réalisent une large intégration interfonctionnelle de capacités partielles (« midrange capabilities »). Ces capacités organisationnelles de haut niveau sont généralement associées à des capacités cognitives individuelles et collectives des membres de l'équipe de direction et plus généralement du sommet de la hiérarchie, ceux qui participent aux conversations stratégiques et aux choix d'organisation. Ainsi les chercheurs ont analysé les cartes mentales des managers (Huff, 1990), les styles cognitifs des membres de la direction (Hurst, Rush et White, 1989), et la complexité cognitive des dirigeants (Calori et al., 1994).

Dans ce chapitre, nous examinons deux nouvelles caractéristiques des structures cognitives des cadres : leur forme de logique préférée (c'est-à-dire leur processus formel de pensée indépendamment de leur contenu) et leur rationalité sociologique (les critères de validité qu'ils mobilisent dans leur communication avec les autres). Ces nouvelles facettes, modes de raisonnement et de communication, paraissent importantes dans une théorie expliquant les modes de coordination et d'intégration de connaissances réparties dans une hiérarchie.

Dans un système de connaissances réparties, le cerveau du stratège ne peut contenir toutes les connaissances nécessaires (Grant, 1996b; Hayek, 1989; Spender, 1996; Tsoukas, 1996). De plus, la création de connaissances est une activité individuelle (Grant, 1996b) et l'apprentissage requiert un engagement personnel dans l'action (Cohen et Sproull, 1996). Pour toutes ces raisons, il semble pertinent d'adopter une perspective élargie et de considérer les caractéristiques cognitives de l'ensemble des membres de l'organisation en interaction les uns avec les autres (pas seulement les dirigeants).

Cependant, s'il s'agit d'intégrer les connaissances spécifiques de plusieurs individus (Grant, 1996a) pour créer de nouvelles combinaisons (Kogut et Zander, 1992), alors les routines et les formes organisationnelles qui définissent les ensembles de relations entre individus jouent un rôle majeur. Donc, ceux qui ont le pouvoir de définir l'organisation formelle et de légitimiser les routines, i.e. 
les dirigeants, ont les rôles principaux en matière d'intégration, et l'ensemble de leurs capacités cognitives semble être la composante principale de la capacité organisationnelle. Selon Grant : «L'architecture des capacités doit correspondre au moins partiellement avec la structure d'autorité de l'entreprise » (Grant, 1996a, p. 378).

Finalement le chercheur qui veut expliquer le développement de la firme par le développement de l'ensemble des connaissances spécifiques qu'elle rassemble, ne peut qu'adopter un sujet ambivalent : l'ensemble des membres de l'organisation, mais avec une attention particulière au sous-ensemble des dirigeants.

Dans un système de connaissances réparties, la connaissance émerge continuellement au niveau individuel et n'est pas complètement identifiable (Tsoukas, 1996), donc l'intégration des connaissances ne peut se réaliser qu'au sein de groupes partiels dans lesquels les flux de connaissances sont fluides (Grant, 1-996b), de façon décentralisée, avant de s'étendre éventuellement à l'ensemble de l'organisation. La dynamique de la création et de l'intégration des connaissances (flux) entre les niveaux individu, groupe et organisation - devient alors un sujet de recherche prioritaire.

\subsection{Perspectives sur l'apprentissage organisationnel}

Pour résumer la théorie de l'apprentissage organisationnel (voir chapitre $5 \mathrm{du}$ présent ouvrage), nous retenons cinq contributions qui se complètent et apparaissent compatibles avec les concepts de logique dialectique et d'agir communicationnel : March (1991), Senge (1992), Weick (1995), Nonaka et Takeuchi (1995), Crossan, Lane et White (1999). D'autres travaux complè- tent ces cadres de référence : sur l'inclusion des cadres intermédiaires ( middle managers ») dans les conversations stratégiques et sur la justice procédurale.

Selon March (1991), le développement et l'utilisation des connaissances dans l'organisation s'expliquent par un double processus d'exploration et d'exploitation. L'exploration de nouvelles possibilités permet l'adaptation dans un univers turbulent et produit ses résultats à long terme. L'exploitation des certitudes et compétences passées (apprentissage du « code organisationnel » par ses membres) produit ses résultats plus rapidement dans un univers relativement stable. L'équilibration entre la capacité d'exploration et la capacité d'exploitation est le fondement de l'apprentissage organisationnel, le principal facteur de survie et de sécurité du système. Les récompenses de l'exploitation sont plus certaines et plus proches dans le temps et l'espace que les récompenses de l'exploration. Pour cette raison la plupart des organisations ont tendance à favoriser l'exploitation au détriment de l'exploration selon March (1991), cette tendance a des conséquences autodestructrices à long terme dans un environnement turbulent.

Plusieurs caractéristiques et pratiques organisationnelles permettent de maintenir un niveau suffisant d'exploration : la rotation des individus («turnover »), le retardement de la socialisation des nouveaux membres, un système de récompenses adéquat, en bref plusieurs pratiques de management des ressources humaines. Levinthal et March (1993) notent également l'influence des préférences et des perceptions face au risque et des processus de sélection de nouvelles pratiques sur la capacité d'exploration, mais ils ne s'attardent pas sur les capacités cognitives individuelles qui peuvent 
sous-tendre ces pratiques. Cette théorie qui aboutit à préserver un certain niveau d'hétérogénéité dans un système, en réconciliant l'exploration et l'exploitation, est construite sur un raisonnement dialectique. Nous ferons plus loin l'hypothèse que les membres de l'organisation qui comprennent le développement selon une dialectique exploration-exploitation sont plus capables de mettre en pratique ces concepts et de vivre ces tensions. Nous suggérerons également l'existence d'une relation entre la dynamique d'exploitation de certitudes et « l'agir stratégique » orientée vers un but auquel tous les membres doivent adhérer (selon Habermas), et l'existence d'une relation entre la dynamique d'exploration de nouvelles idées et l'agir communicationnel où les uns s'ouvrent aux idées des autres (selon Habermas).

Dans la « Cinquième Discipline », celle de l'apprentissage organisationnel, Senge (1992) souligne l'importance des caractéristiques cognitives des membres de l'organisation : maîtrise personnelle (vision, tension créatrice, intuition), schémas mentaux (capacité de réflexion), ouverture transcendant les jeux politiques internes et l'intérêt personnel, respect de la diversité et des solutions locales, et apprentissage au sein d'une 'équipe par la pratique de la discussion et du dialogue. Le dialogue est un nécessaire complément aux discussions :

«La discipline de l'apprentissage en équipe commence avec le «dialogue »-, la capacité des membres de l'équipe à suspendre leurs croyances et à entrer dans une pure « réflexion ensemble». Le « dialogue » est différent de la « discussion » plus courante, qui littéralement est « un tiraillement d'idées dans une compétition où le gagnant rafle toute la mise » (Senge, 1992, p. 10).
Comme le souligne Senge, le développement de dialogues est souvent empêché par des « routines défensives » (Argyris, 1985). D'après Argyris, l'origine des routines de défense n'est ni la croyance ferme en nos idées ni le désir de préserver nos relations sociales, c'est la peur d'exposer la réflexion qui se cache derrière nos idées. Ainsi les routines défensives empêchent de remettre en question la logique de notre pensée et la validité de nos arguments dans une communication.

La théorie de l'agir communicationnel apparaît alors comme le fondement philosophique et sociologique des concepts mis en avant par Senge. La rationalité « d'agir communicationnel » selon Habermas fonde la pratique du dialogue : « un flux de sens qui passe librement entre les personnes »...» On essaie pas de gagner dans un dialogue $» .$. « les personnes vont au-delà de leur compréhension individuelle $» .$. « elles deviennent observateurs de leur propre pensée $» \ldots$ « elles suspendent leurs postulats »... « elles s'aident mutuellement à découvrir les incohérences dans leurs raisonnements respectifs » (Senge, 1992).

Le processus de «construction de sens » (sensemaking) d'après Weick (1995) est également un processus socio-cognitif auquel participent des managers « auteurs » et « historiens » qui comprennent le mouvement perpétuel (Weick, 1995, p. 187-188) et la durée, et qui se communiquent leurs interrogations en se rencontrant. Les réunions ne doivent pas être des lieux d'alignement cognitif mais plutôt des lieux de conversation sociale : «Les réunions sont des constructions de sens. Elles donnent corps à l'organisation et constituent sa substance. Elles sont aussi l'un des lieux principaux où la variété requise peut être mobilisée dans 
l'intérêt de comprendre et de réguler un peu de la variété à laquelle l'organisation est confrontée » (Weick, 1995, p. 187).

L'originalité de la position de Weick réside dans la primauté donnée à l'action dans le processus d'apprentissage. L'apprentissage est essentiellement rétrospectif, après l'action, dans le monde vécu (Weick, 1995, p. 182). L'intercompréhension mutuelle est donc fondée sur des expériences partagées : «Si le sens est tiré de l'action, alors les sens différents peuvent être équivalents sans pour cela être similaires. Si j'agis en fonction de ma compréhension d'une expérience commune et si tu agis en fonction de ta compréhension différente de la même expérience, nous restons reliés ensemble par l'origine commune de nos compréhensions [...] Cette communauté d'expérience nous relie et rend possible à chacun d'entre nous la compréhension du sens que l'autre a construit » (Weick, 1995, p. 189). Ce principe traduit la rationalité d'agir communicationnel fondée par des « actions coordonnées dans le monde vécu » qui permettent l'intersubjectivité.

Nonaka et Takeuchi (1995) reconnaissent le dialogue et l'expérience partagée comme les moteurs de la création de connaissances organisationnelles. La complémentarité de ces pratiques est expliquée par la nature des connaissances : certaines sont tacites (développées dans l'action et difficilement exprimables), d'autres sont explicites (verbalisables et communicables par la conversation). L'explicitation au moins partielle de la connaissance tacite est la clé de l'apprentissage organisationnel. La « socialisation » établit un champ d'interactions entre individus qui partagent la même expérience génératrice d'une connaissance tacite. «L'externalisation » rend explicite une partie de ces connaissances par « le dialogue et la réflexion collective » (Nonaka et Takeuchi, 1995, p. 71). Puis la combinaison par communication de multiples connaissances explicites transmet la connaissance à d'autres parties de l'organisation par un effet de réseau. Enfin l'apprentissage dans l'action permet l'internalisation de la connaissance par chaque nouvel individu concerné. La rationalité d'agir communicationnel offre également une base philosophique à ce mouvement spiralaire du tacite à l'explicite et de l'individu au collectif. Chez Nonaka et Takeuchi cependant, la dynamique du passage du niveau individuel au niveau organisationnel apparaît comme un processus continu et relativement consensuel. L'explication de ce passage, proposée par Crossan, Lane et White (1999), est plus instructive, car elle admet des processus différenciés suivant les niveaux et des tensions dialectiques.

Le modèle proposé par Crossan, Lane et White (1999), considère que les tensions entre la connaissance institutionnalisée du passé et le besoin de légitimation des nouvelles connaissances sont au cœur du processus d'institutionnalisation de l'innovation au niveau de l'ensemble de l'entreprise (cf. March, 1991). Le modèle est composé de trois niveaux : (1) individuel, (2) groupe, (3) organisation; et de quatre processus psychologiques et sociologiques principaux : l'intuition (au niveau 1), l'interprétation (aux niveaux 1 et 2), l'intégration (aux niveaux 2 et 3), et l'institutionnalisation (au niveau 3). Les idées nouvelles ne viennent, par intuition, qu'aux individus. L'interprétation est l'explication d'une idée à soi-même et à d'autres, par des mots et/ou des actes, ces conversations peuvent renforcer l'idée et le projet. L'intégration est « le 
processus de développement d'une compréhension partagée par les individus et de passage à l'action coordonnée par ajustement mutuel. Le dialogue et l'action en commun sont essentiels pour développer une compréhension mutuelle » (Crossan et al., 1999 p. 525). Puis, si les résultats sont favorables, les idées-actions sont institutionnalisées et deviennent des routines. L'ensemble du processus se déroule en spirales d'exploration et d'exploitation (March, 1991). Les phases d'exploitation correspondent à des moments où le bien-fondé de la stratégie est démontré à l'ensemble des membres de l'organisation pour les convaincre, selon les principes de l'agir stratégique et de la logique binaire (le vrai et le faux) et pour faciliter ainsi la mise en œuvre du niveau organisationnel (représenté par le sommet de la hiérarchie) au niveau individuel. Les phases d'exploration correspondent à des moments de création puis d'interprétation, d'intégration et de légitimisation, du niveau individuel au niveau organisationnel (représenté par le sommet de la hiérarchie). Encore faut-il que les membres du sommet de la hiérarchie soient prêts à (1) écouter les contradictions et à comprendre les arguments exprimés à d'autres niveaux de la pyramide hiérarchique et (2) respecter certains principes de justice procédurale.

L'inclusion des cadres intermédiaires dans les conversations stratégiques touche à la répartition du pouvoir dans l'organisation, qui peut être conçue comme un jeu à somme nulle ou un jeu à somme positive. Les cadres intermédiaires sont en compétition pour « vendre leurs idées au top management ». Selon Dutton et Ashford (1993), la vente de problèmes et d'idées ( «issues ») est considérée comme une activité critique dans les premières phases des décisions concernant l'organisation. Le processus par lequel une question devient stratégique (et compose la partie émergente de la stratégie) est essentiellement rhétorique : les idées sont véhiculées par des démonstrations et argumentations, elles montent dans la hiérarchie quand la rhétorique est efficace, et quand l'autre écoute.

Dans une relation asymétrique entre un cadre « intermédiaire » et un cadre « supérieur », la capacité d'écoute du supérieur est une condition nécessaire pour la progression d'arguments valides. Même dans les cas, de plus en plus fréquents, où les dirigeants ont institutionnalisé l'inclusion des cadres intermédiaires dans les conversations stratégiques, la génération et la progression d'idées nouvelles sont souvent étouffées par des incapacités communicationnelles. Westley (1990) a étudié des interactions verbales concernant des questions stratégiques dans des relations supérieur-subordonné. Une analyse approfondie des conversations montre que, souvent, le supérieur hiérarchique entend mais n'écoute pas vraiment l'autre et, consciemment ou inconsciemment, volontairement ou involontairement, ferme l'argumentation. Lorsque le subordonné est dominé dans la conversation, le mouvement dialectique ne peut pas se développer, les arguments ne s'enchaffient pas et les possibilités de dialogue sont inexistantes (Westley, 1990). De telles discussions asymétriques se terminent toujours par la victoire de l'un contre l'autre, la victoire de celui qui a le pouvoir formel de définir les règles (le cadre rationnel et émotionnel) de la discussion. En bref Westley nous met en garde contre la fausse participation des cadres intermédiaires, qui ne produit que des frustrations.

Ceci nous conduit à une autre voie explorée par un petit nombre de chercheurs en mana- 
gement stratégique : la justice dans la définition des règles de communication entre supérieurs et subordonnés, centre et périphérie. Cet aspect a été traité au chapitre 6 du présent ouvrage. Il suffit de rappeler ici que la capacité de contester et de réfuter est fortement et positivement liée à la capacité de renouveau stratégique de l'organisation (Kim et Mauborgne, 1995). On touche ici à des principes fondamentaux de la rhétorique.

\subsection{Rhétorique, dialogue et apprentissage}

Dans le schéma théorique que nous construisons, les pratiques réelles de communication, rhétorique et/ou dialogue, constituent le troisième niveau, a priori plus facile à observer que la rationalité sociologique et la logique des individus. Certains chercheurs en management stratégique et développement des organisations ont exploré les concepts de la rhétorique définis à l'origine par la philosophie et repris par la science politique. Dans le cadre d'une organisation orientée vers des buts il s'agit de la rhétorique « délibérative » selon Aristote (édition de 1991) : ceux qui doivent décider un ensemble d'actions dans le futur construisent des arguments contraires leur permettant de progresser vers une décision pour le bien de la collectivité. Les derniers mots de cette définition sont essentiels : « pour le bien de la collectivité », sinon comment comprendre qu'Aristote (d'abord formé à l'Académie contre les dangers de la rhétorique) ait composé une œuvre majeure sur le sujet. Le rhétoricien doit être sincère, sage et viser le bien commun.

La rhétorique délibérative formule des prédictions sur les résultats escomptés qui doivent être utiles et sans danger. Donc les conclusions du discours peuvent être testées a posteriori, le rhétoricien et l'audience doivent alors s'intéresser à la réalité qui se déroule. « La rhétorique se rattache à la dialectique » (Rhétorique, Livre premier, Chapitre premier, première phase : Aristote, édition de 1991, p. 75). La dialectique est une technique de raisonnement principielle, alors que la rhétorique s'efforce de résoudre des questions particulières.

Dans un processus de délibération, le rhétoricien comme le dialecticien doivent être capables de construire des arguments contraires.

La « Nouvelle Rhétorique » (Perelman, 1977, 1982) prolonge les principes Aristotéliciens de délibération : elle apporte une distinction pertinente entre deux types de processus rhétoriques, la démonstration vs l'argumentation. Dans la « démonstration », les acteurs défendent leurs positions avec un minimum de changements, ils répètent leurs arguments, ils se réfèrent à des vérités, s'appuient sur la rationalité, des prémisses aux conclusions, et fondent leurs arguments sur des évidences (Perelman, 1977, 1982). Il faut noter la correspondance entre le concept « d'agir stratégique » défini par Habermas (section 2) et la pratique de la rhétorique démonstrative définie par Perelman. En revanche, dans « l'argumentation » les acteurs gardent leur point de vue ouvert, laissent leur position évoluer au fil du temps, s'accommodent des contradictions en découplant les problèmes, utilisent la durée du processus, inventent de nouveaux arguments en chemin, se réfèrent à des opinions, des justifications et des critiques, considèrent les contingences des conditions individuelles, sociales et historiques, adoptent un processus ouvert, et s'appuient sur des évidences (Perelman, 1977). L'argumentation ainsi définie est proche de la délibération et semble être la manifestation d'un agir communicationnel. Corvellec (2000) fournit plusieurs exemples 
utiles des deux types de rhétorique dans le déroulement d'un projet. L'étude approfondie de cas montre cependant que la frontière entre démonstration et argumentation n'est pas toujours clairement tracée.

Müllern, Stein et Melin (1997) offrent une distinction plus subtile entre des expressions rhétoriques « ouvertes » ou « fermées ». Dimension fondamentale du processus de communication, le « degré de fermeture » différencie « l'argumentation fermée » de « l'argumentation ouverte ». «L'argumentation fermée » est parente de la démonstration définie par Perelman, bien qu'elle ne prenne pas la même forme (une approche critique la considérerait comme une forme cachée de démonstration). Dans la vie des affaires, l'argumentation fermée est un moyen de diffuser et de protéger l'idéologie managériale dominante. Elle s'appuie sur diverses tactiques de fermeture de l'échange d'arguments : la définition de l'ordre du jour, la logique propositionnelle, le recours à l'évidence, la confusion entre les faits et l'idéologie, l'exagération, la répétition, la preuve sociale, la référence au pouvoir, la cooptation, la référence à des textes, et diverses actions non verbales (Müllern et al., 1997). La longueur de cette liste montre le caractère politique et le défi éthique de la vraie rhétorique délibérative dans les organisations, Aristote doit se retourner dans sa tombe ! Lorsque le caractère manipulatif de l'argumentation fermée est révélé, elle peut produire des résistances passives ou actives. Sinon, lorsque le bien collectif est visé et que la hiérarchie est respectée, elle peut accélérer le passage à l'action et donc l'exploitation des opportunités. « L'argumentation ouverte » crée l'ambiguïté et stimule des interprétations multiples, en progressant vers une construction de sens collective. Müllern et al. (1997) décrivent l'argumentation ouverte comme un processus de changement à plusieurs voix. Elle revêt toutes les caractéristiques de la vraie délibération selon Aristote, et apparaît comme une manifestation concrète d'un agir communicationnel orienté vers le succès collectif. En relation avec les conditions de symétrie posées par Habermas (section 2) l'argumentation ouverte est « polycentrique », alors que l'argumentation fermée et la démonstration sont "égocentriques ». D'après Moscovici et Doise (1994), dans un groupe social, la construction collective de consensus doit explorer et vivre des conflits. Les conflits et les critiques ne doivent pas être évités et les coalitions les plus extrêmes doivent être invitées au débat. Sinon, si elles sont exclues des discussions, il existe un risque de polarisation du groupe vers l'un des extrêmes. En d'autres termes la dynamique de l'opposition entre les extrêmes semble produire de meilleurs résultats qu'une stratégie de conformation basée sur la recherche de plus d'information et la croyance a priori en un compromis à mi-chemin (Moscovici et Doise, 1994). Au niveau individuel et au niveau organisationnel, Sfez (1988) annonce les dangers de la réception passive et du conformisme. Il définit le «tautisme » et condamne le pouvoir « tautistique » : « Ici la communication n'est plus que la répétition imperturbable du même (tautologie) dans le silence d'un sujet-mort ou sourd-muet, enfermé dans sa forteresse intérieure (autisme), capté par un grand Tout qui l'englobe et dissout jusqu'au moindre de ses atomes paradoxaux. Cette totalité sans hiérarchie, cet autisme tautologique je les nomme tautisme, néologisme qui condense totalité, autisme et tautologie » (Sfez, 1988, p. 92). 
Finalement nous retiendrons trois types d'expressions rhétoriques composant les discussions : la démonstration, l'argumentation fermée et la délibération (ou argumentation ouverte). La délibération se différencie nettement des deux premiers types, dans la mesure où elle est polycentrique. En ce sens, elle est proche du dialogue qui est une forme de communication extérieure à la rhétorique.

« Le dialogue », comme nous l'avons vu avec Senge, n'a pas pour but de convaincre et n'a pas pour but premier d'aboutir à des décisions collectives. Les termes «d'émetteur » de « récepteur » et «d'audience» si chers à la littérature des affaires sont bannis, car le but essentiel du dialogue est la compréhension intersubjective. Nous ne répéterons pas ici la description des principes et des processus de dialogue (voir section 2) qui apparaissent comme la manifestation concrète de rationalités d'agir communicationnel. Dans la mesure où les compréhensions individuelles parfois opposées sont respectées et écoutées par l'autre tour à tour, on trouve dans un dialogue des traces de logique hyperdialectique (voir section 1). Au niveau individuel Riegel (1976) considère que le développement humain prend sa source dans des interactions « dialogiques » caractérisées par des relations symétriques et des dialogues synchronisés (alors que dans la petite enfance la relation mère-enfant est asymétrique et dans la vieillesse les dialogues ont tendance à dégénérer en alternance de monologues). Au niveau organisationnel, dans l'idéal-type «post bureaucratique » le dialogue est institutionnalisé (Heckscher, 1994), les interactions sont fondées sur l'influence et la confiance plus que sur les relations de pouvoir, ainsi les décisions tendent à fluctuer lorsque de nouveaux dialogues s'instaurent. Les contributions majeures à la théorie de l'apprentissage organisationnel (voir section 3.2.) accordent au dialogue une fonction fondamentale dans tout processus d'exploration. Cependant, s'il fallait faire un bilan général des incursions du management dans l'univers des discours et conversations stratégiques on découvrirait probablement que le courant orthodoxe dominant du management stratégique (voir Barry et Helmes, 1997; Ford et Ford, 1995) s'intéresse principalement à la rhétorique au service de la conduite du changement par le haut de la hiérarchie, fondée sur la persuasion des membres de l'organisation chargés de la mise-en-œuvre des décisions stratégiques, selon une rationalité d'agir stratégique qui a la certitude binaire de détenir la vérité.

\section{Les logiques et les rationalités sociologiques, racines de l'apprentissage et du développement organisationnel}

L'analyse des théories de l'apprentissage expliquant le développement organisationnel (section 3) révèle un accord sur plusieurs points :

- le caractère dialectique de l'apprentissage organisationnel dans un mouvement d'exploration-exploitation;

- le déficit d'exploration dans la plupart des organisations

- l'expérience vécue en commun comme source de création et diffusion de connaissances tacites;

- le dialogue comme forme de communication nécessaire à l'apprentissage exploratoire; la délibération (ou argumentation ouverte) comme forme de progression vers un accord, le mouvement spiralaire de création et de diffusion des connaissances nouvelles, 


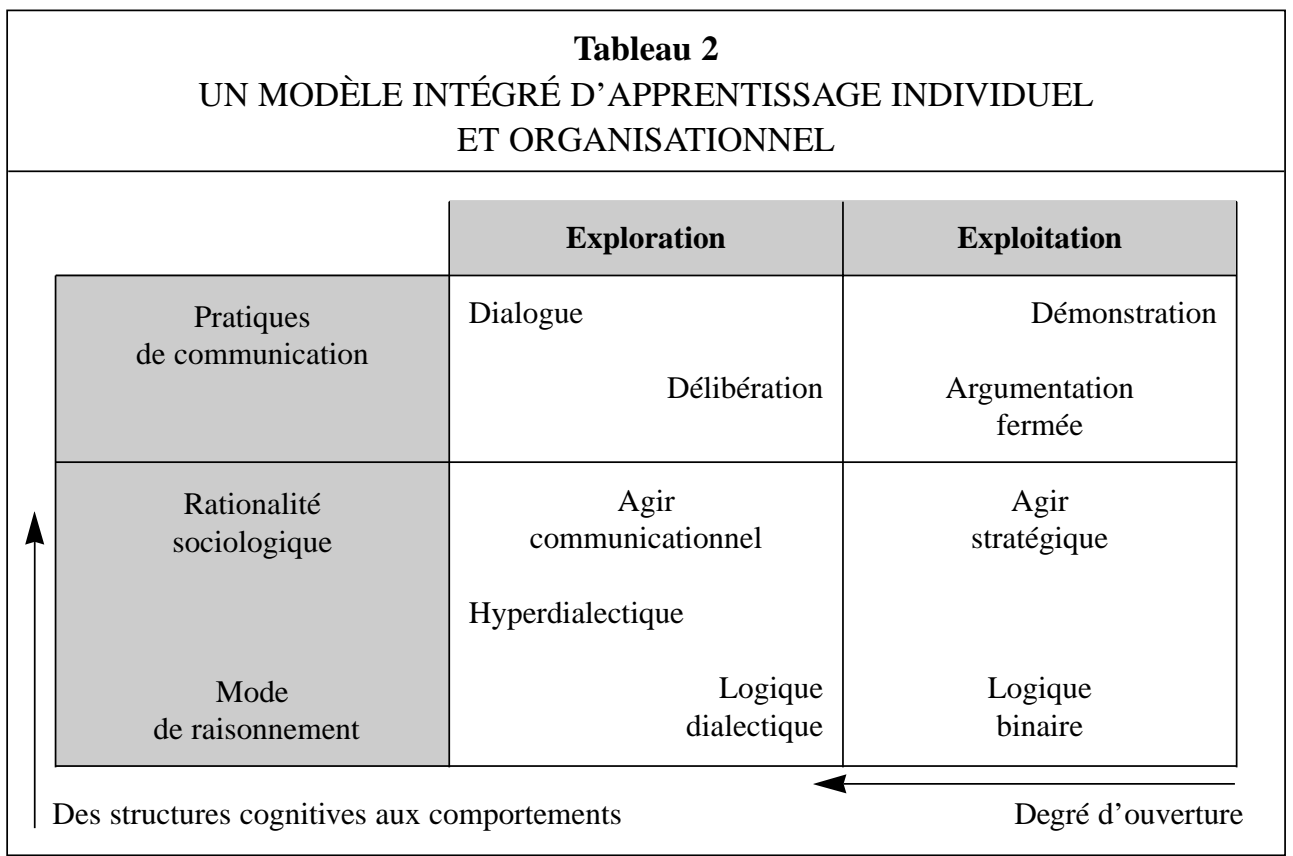

du niveau individuel au niveau organisationnel (exploration) et dans l'autre sens (exploitation), par des processus complémentaires (intuition, interprétation, intégration, légitimisation).

On distingue quatre formes pratiques de communication : le dialogue et trois formes de rhétorique - la délibération, l'argumentation fermée et la démonstration.

En mobilisant les principes de la logique binaire, de la logique dialectique (Hegel) et des métalogiques hyperdialectiques (Gurvitch, Merleau Ponty), notre objectif était de découvrir les racines cognitives des rationalités sociologiques d'acteurs. Les liens entre chaque type de logique et chaque type de rationalité sociologique ont été mis en évidence (section 2).

En mobilisant la théorie de l'agir communicationnel selon Habermas, notre objectif était de découvrir les racines cognitives des pratiques de communication et des capacités individuelles et organisationnelles d'exploration et d'exploitation. Deux rationalités sociologiques ont été ainsi mises à jour : l'agir stratégique et l'agir communicationnel, et les liens entre chacune de ces rationalités et les pratiques de communication ont été mis en évidence (section 3.2.).

Ces concepts et leurs liens sont résumés dans le tableau 2.

Des structures cognitives aux comportements Le tableau 2 schématise les deux niveaux de notre modèle intégrateur :

(1) Formes et pratiques de communication (pratiques socio-cognitives généralement observables).

(2) Structures socio-cognitives : rationalités sociologiques et raisonnements logiques (plus difficilement observables).

Le côté droit du tableau décrit l'ensemble des concepts et pratiques qui favorisent l'ex- 
ploitation (logique binaire, rationalité d'agir stratégique, démonstration et argumentation fermée). Le côté gauche dû tableau décrit l'ensemble des concepts et pratiques qui favorisent l'exploration (hyperdialectique et logique dialectique, rationalité d'agir communicationnel, délibération et dialogue). De droite à gauche le tableau est construit selon un axe horizontal qui représente le degré d'ouverture à la pensée de l'autre.

Les liens entre concepts/pratiques favorisant l'exploration d'une part et l'exploitation d'autre part, (préalablement discutées en sections 2 et 3), peuvent être ainsi résumés : - la capacité et la pratique de dialogue et de délibération d'un individu (et d'une organisation) sont liées à sa rationalité sociologique d'agir communicationnel; inversement, la capacité et la pratique de la démonstration et de l'argumentation fermée sont liées à une rationalité d'agir stratégique; - la rationalité sociologique d'agir communicationnel d'un individu (et d'une organisation) est liée à sa forme de raisonnement logique préférée : hyperdialectique et/ou dialectique; inversement la rationalité sociologique d'agir stratégique est liée à la forme de logique préférée : logique binaire. Comme nous l'avons mentionné précédemment, il est possible d'observer et d'analyser les pratiques et préférences d'un individu en matière de communication (premier niveau), mais il est également possible d'analyser la rationalité sociologique adoptée par un individu (analyse de discours et de conversations) et d'analyser sa forme de raisonnement logique préférée. Ainsi les propositions d'étapes que nous formulons ici pourraient faire l'objet de recherches empiriques futures.

Les discussions des sections précédentes nous conduisent également à formuler plu- sieurs explications de la capacité d'apprentissage organisationnel - exploration vs exploitation - par les capacités et pratiques de communication, les rationalités sociologiques et les capacités de raisonnement logique :

- la capacité d'apprentissage d'une organisation, plus précisément sa capacité d'exploration, est liée positivement aux capacités et pratiques de dialogue et de délibération de l'ensemble de ses membres, notamment de ses dirigeants;

- la capacité d'exploitation d'une organisation est liée positivement aux capacités et pratiques de démonstration et d'argumentation fermée de l'ensemble de ses membres, notamment de ses dirigeants.

Les relations entre les capacités d'apprentissage et les rationalités sociologiques sont médiatisées par les pratiques de communication, elles peuvent être résumées ainsi :

- la capacité d'apprentissage d'une organisation, plus précisément sa capacité d'exploration, est liée positivement aux capacités rationnelles sociologiques « d'agir communicationnel » de l'ensemble de ses membres, notamment de ses dirigeants;

- la capacité d'exploitation d'une organisation est liée positivement aux capacités rationnelles sociologiques « d'agir stratégique » de l'ensemble de ses membres, notamment de ses dirigeants.

Les relations entre les capacités d'apprentissage et les formes de raisonnement logique sont médiatisées par les pratiques de communication et par les rationalités sociologiques, elles peuvent être résumées ainsi :

- la capacité d'apprentissage d'une organisation, plus précisément sa capacité d'exploration, est liée positivement aux capacités logiques, dialectique ouverte et hyperdialectique, de l'ensemble de ses membres, notamment de ses dirigeants; 
- la capacité d'exploitation d'une organisation est liée positivement aux capacités logiques, logique binaire, de l'ensemble de ses membres, notamment de ses dirigeants. Jusqu'ici les lecteurs préoccupés prioritairement par les nouvelles théories du développement organisationnel ont pu être gênés par l'ambiguïté entretenue dans ce chapitre entre le niveau individuel et le niveau collectif (organisationnel). Notamment la référence à des structures cognitives par nature individuelles a pu faire croire à un glissement vers la psychologie des managers, au détriment des thèses économiques et sociologiques s'appliquant au niveau de l'entreprise. Il est temps de confirmer que cette ambiguité et l'attention portée aux capacités cognitives des individus membres de l'organisation, notamment les dirigeants, sont délibérées et, à notre sens, ne nous écartent pas de la compréhension de phénomènes de développement organisationnel.

Premièrement, les sources d'inspiration philosophique que nous avons utilisées, comme toute réflexion philosophique, ne se préoccupent pas des différences de niveaux. Leur caractère abstrait, purement conceptuel, offre une compréhension de l'individu et de la collectivité, c'est d'ailleurs ce qui fait l'intérêt d'un étirement de la recherche en gestion vers la philosophie.

Deuxièmement, nous reconnaissons volontiers notre préférence pour une approche des phénomènes organisationnels, caractérisée par l'individualisme méthodologique. L'individualisme méthodologique est un courant de la sociologie qui minimise les déterminismes structurels et pose pour principe initial que les analyses sociologiques doivent prendre l'individu pour objet premier d'observation et comme unité de référence (Boudon et Bourricaud, 1982). Cette perspective est controversée en sociologie générale, mais elle paraît réaliste dans le contexte organisationnel (entreprises) où certains individus (les dirigeants) détiennent une autorité formelle qui leur permet de définir et de légitimer explicitement et implicitement les modalités d'interactions sociales. Dans ces situations économiques et sociales, l'ensemble des structures cognitives individuelles des dirigeants et cadres façonnent les structures formelles et informelles de communication caractérisant l'organisation. Au sommet extrême de la hiérarchie, les capacités et préférences cognitives du « président directeur » façonnent la composition de la coalition dominante et les processus d'interaction entre ses membres. Troisièmement le processus de sélection d'individus et d'accumulation de leurs capacités individuelles peut créer progressivement un effet de masse critique qui confère à l'organisation une caractéristique socio-cognitive, un style particulier incarné par la majorité de ses membres les plus influents.

En d'autres termes, nous revendiquons sans plus d'ambiguïté le statut de théorie (partielle) du développement organisationnel pour le cadre conceptuel développé dans ce chapitre.

La distinction entre le niveau individuel et le niveau organisationnel est cependant particulièrement pertinente pour répondre à la question de la nécessaire coexistence (en équilibre instable) de capacités d'exploration et de capacité d'exploitation au sein d'une même entreprise (March, 1991).

$\mathrm{Au}$ niveau organisationnel, on conçoit la possibilité de cette coexistence grâce à la répartition des capacités individuelles : certains membres ont les capacités requises pour l'exploration, d'autres les capacités 
requises pour l'exploitation. Mais la question se pose au niveau individuel : un même individu peut-il développer de grandes capacités de logique dialectique, une rationalité d'agir communicationnel et une capacité de dialogue et d'argumentation ouverte d'une part et d'autre part des capacités de logique binaire, d'agir stratégique et de démonstration? La réponse à cette question devra attendre des tests empiriques ultérieurs. Nous suggérons cependant qu'il est difficile à un individu de cumuler de grandes qualités dans les deux facettes de profil cognitif. Cela semble possible pour ceux qui ont développé les qualités correspondant à l'agir communicationnel (car ils raisonnent déjà en référence à tous les mondes, dont le monde objectif). En revanche ceux dont les capacités et les préférences sont marquées par la logique binaire, l'agir stratégique et la démonstration pourraient avoir des difficultés à remettre en question leurs certitudes et réduire leur ego. Il semble que le passage d'un mode de raisonnement, de rationalité et de communication à un autre (en fonction des moments, des circonstances et de l'avancement des débats) soit fondé sur une intuition et un ressenti du niveau d'intersubjectivité.

Sur le plan pratique ces considérations nous amènent à plusieurs remarques.

Pour être efficace dans une organisation, un individu devrait avoir conscience de la nature de ses pratiques communicationnelles (dont ses biais), de sa rationalité sociologique et de sa forme de raisonnement logique préférée. Idéalement il devrait aussi percevoir les préférences cognitives de ses principaux interlocuteurs, afin d'enrichir et d'équilibrer les pratiques de relations.

La formation d'équipes devrait tenir compte de ces paramètres cognitifs individuels afin de créer une complémentarité nécessaire au double exercice de l'exploration-exploitation. Selon ce principe également, au niveau d'une direction de projet par exemple, en l'absence d'un leader réunissant toutes les capacités cognitives et communicationnelles, la formation d'un tandem composé d'un « explorateur » et d'un « exploiteur» peut stimuler la dynamique d'apprentissage et de développement.

L'explorateur ainsi que l'entreprise exploratrice auront la sagesse de douter parfois de leurs certitudes stratégiques et de respecter la diversité et les contradictions. On touche ici à la dimension éthique et émotionnelle des interactions (Fay, 1999). Il paraît impossible à une organisation et à des individus qui tolèrent l'injustice et dont tous les actes sont guidés par un ego disproportionné, de se plier à une rationalité d'agir communicationnel et à la pratique du dialogue et de la délibération. La relation entre « ego et alter » est enracinée dans le conscient et dans l'inconscient d'une personne (et dans l'imaginaire d'un groupe social par rapport aux autres groupes), donc probablement moins malléable que ses capacités de raisonnement logique. Il pourrait s'agir d'un troisième niveau à ajouter au modèle intégrateur que nous avons construit, l'ouverture à l'autre produit l'énergie et la patience nécessaires pour ouvrir des dialogues visant la compréhension intersubjective et les délibérations visant le bien de la collectivité. 


\section{BibLIOGRAPHIE}

Adorno T., Against Epistemology : A Metacritique, Cambridge, MA, MIT, 1984.

Argyris C., Reasoning, Learning and Action : Individual and Organizational, San Francisco, Jossey Bass, 1982.

Argyris C., Strategy, Change and Defensive Routines, Boston, MA, Pitman, 1985.

Aristotle, The Art of Rhetoric, London, Penguin Books, 6OBC, 1991.

Barry D. et Elmes M., « Strategy Retold : Toward a Narrative View of Strategic Discourse », Academy of Management Review, 22 (2), 1997, p. 429-452.

Benson J.K., « Organizations : A Dialectical View », Administrative Science Quarterly, 22, 1, 1977, p. 1-21.

Boudon R. et Bourricaud F., Dictionnaire critique de la sociologie, Paris, PUF, 1982.

Burbidge J.W., « Hegel's Conception of Logic » in F.B. Beiser (Eds.), The Cambridge Companion to Hegel, Cambridge UK, Cambridge University Press, 1993, p. 86-101.

Calori R., « Organizational Development, Toward Creative Dialectical Evolution », Organization, Special Issue, 9 (1), 2002.

Calori R., Johnson G. et Sarnin P., « CEO’s Cognitive Maps and the Scope of the Organization », Strategic Management Journal, 15 (6), 1994, p. 437-457.

Carr A., "In Search of the Other : The "Separation Thesis" and the Relevance of its Psychodynamics to Work Organizations ", 17 ${ }^{\text {th }}$ EGOS Colloquium, Lyon, France, 5-7 July 2001.

Cohen M.D. et Sproull L.S., (Eds.), Organizational learning, Thousand Oaks CA, Sage Publications, 1996.

Corvellec H., "Talks on Tracks, Debating Urban Infrastructure Projects », Gothenburg Research Institute, Report 2000-8, 2000.

Crossan M.M., Lane H.W. et White R.E., « An Organizational Learning Framework : From Intuition to Institution », Academy of Management Review, 24 (3), 1999, p. 522-537.

Dutton J.E. et Ashford S.J., « Selling Issues to Top Management », Academy of Management Review, 18 (3), 1993, p. 397-428.

Evans P. et Doz Y.L., « The Dualistic Organization » in P. Evans, Y.L. Doz et A. Laurent, Human Resource Management in International Firms, London, Mcmillan Press, 1989, p. 219-245.

Fay E., « Du traitement de l'information au commerce de la parole, pour un management délibératif des processus ouverts dotés de réseaux informatiques », Thèses de Doctorat en Sciences de Gestion, IAE Université de Lyon 3, Lyon, 1999.

Ford J.D. et Backhoff R., « Organizational Change in and out of Dualities and Paradox » in R. Quinn et K. Cameron (Eds.), Paradox and Transformation: Toward a Theory of Change in Organization and Management, Cambridge, MA, Ballinger, 1988, p. 81-121.

Ford J.D. et Ford L.W., " Logics of Identity, Contradiction and Attraction in Change », Academy of Management Review, 19 (4), 1994, p. 756-785.

Ford J.D. et Ford L.W., « The Role of Conversations in Producing Intentional Change in Organizations », Academy of Management Review, 20 (3), 1995, p. 541-570. 
Forster M., « Hegel's Dialectical Method » in F.C. Beiser, The Cambridge Companion to Hegel, Cambridge, UK, Cambridge University Press, 1993, p. 130-170.

Grant R.M., « Prospering in Dynamically-Competitive Environments : Organization Capability as Knowledge Integration », Organization Science, 7, 1996a, p. 375-387.

Grant R., « Toward a Knowledge-Based Theory of the Firm », Strategic Management Journal, 17, Special Issue, Winter 1996b, p. 109-122.

Gurvitch G., Dialectique et Sociologie, Paris, Flammarion, 1962.

Habermas J., The Theory of Communicative Action, volume 1 : Reason and the Rationalization of Society, Boston, MA, Beacon Press, 1981, 1984.

Habermas J., Théorie de l'agir communicationnel, tome 1 : rationalité de l'agir et rationalisation de la société, Paris, Fayard, 1981, 1987.

Hampden Turner C., Charting the Corporate Mind, from Dilemma to Strategy, Oxford, Basil Blackwell, 1990.

Hannan M. et Freeman J., «The Population Ecology of Organizations », American Journal of Sociology, 82, 1977, p. 929-964.

Hayek F.A., « The Pretense of Knowledge », American Economic Review, 79, 1989, p. 3-7. Heckscher C., « Defining the Post-Bureaucratic Type », in C. Heckscher et A. Donnellon (éds.), The Post-Bureaucratic Organization, Thousand Oaks, CA, Sage Publications, 1994. Hedberg B.L.T., Nystrôm P.C. et Starbuck W.H., " Camping on Seesaws : Prescriptions for a Self-Designing Organization », Administrative Science Quarterly, 21, 1976, p. 41-64.

Hegel G.W.F., Science de la logique, Tome I, Paris, Aubier, 1812, 1972.

Hegel G.W.F., Science de la logique, Tome II, Paris, Aubier, 1812, 1981.

Hegel G.W.F., Science of Logic, Atlantic Highlands, NJ, Humanities Press International, 1816, 1989 édition.

Huff A.S., Mapping Strategic Thought, Chichester, UK, Wiley, 1990.

Hurst D.K., Rush J.C. et White R.E., « Top Management Teams and Organizational Renewal », Strategic Management Journal, 10 (4), 1989, p. 87-105.

Kim W.C. et Mauborgne R.A., «A Procedural Justice Model of Strategic Decision Making : Strategy Content Implications in the Multinational », Organization Science, 6 (1), 1995, p. 44-61.

Kogut B. et Zander U., « Knowledge of the Firm, Combinative Capabilities, and the Replication of Technology », Organization Science, 3, 1992, p. 383-397.

Levinthal D.A. et March J.G., « The Myopia of Learning », Strategic Management Journal, 14, Special Issue, Winter 1993, p. 95-112.

March J.G., Decisions and Organizations, Oxford, Blackwell, 1988.

March J.G., « Exploration and Exploitation in Organizational Learning », Organization Science, 2, 1991, p. 71-87.

Merleau-Ponty M., Le visible et l'invisible, Paris, Gallimard, 1964.

Miller D., The Icarus Paradox, New York, Harper Business, 1990.

Mintzberg H. et Waters J.A., « Of Strategies, Deliberate and Emergent », Strategic Management Journal, 6 (3), 1985, p. 257-272. 
Modell A., The Private Self, Cambridge, MA, Harvard University Press, 1996.

Morin E., La Méthode (4) Les Idées, Paris, Editions du Seuil, 1991.

Moscovici S. et Doise W., Conflict and Consensus, A General Theory of Collective Decisions, London, Sage Publications, 1994.

Müllern T., Stein J. et Melin L., « On the Rhetoric of Managing Change : The Initial Phase », EIASM Conference, Leuven, Belgium, 4-6 June 1997.

Nonaka I. et Takeuchi H., The Knowledge-Creating Company, New York, Oxford University Press, 1995.

Perelman C., The Realm of Rhetoric, Notre Dame (IN) and London, University of Notre Dame Press, 1977, 1982.

Piaget J., Introduction à l'épistémologie génétique, Paris, PUF, 1972.

Prahalad C.K. et Doz Y.L., The Multinational Mission : Balancing Local Demands and Global Vision, New York, The Free Press, 1987.

Riegel K.F., « The Dialectics of Human Development », American Psychologie, October 1976, p. 689-700.

Samra-Fredericks D., « Talking of Emotion for the Development of Strategy in, the Boardroom », $2^{\text {nd }}$ Critical Management Studies Conference, Manchester, 11-13 July 2001.

Schumpeter J., The Theory of Economic Development, Cambridge, Mass., Harvard University Press, 1934, 1989.

Senge P.M., The Fifth Discipline, the Art and Practice of the Leaming Organization, London, Century Business, 1992.

Sfez L., Critique de la Communication, Paris, Éditions du Seuil, 1988.

Spender J.-C., « Making Knowledge the Basis of a Dynamic Theory of the Firm », Strategic Management Journal, 17, Special Issue, Winter 1996, p. 45-62.

Toulmin S., Rieke A. et Janik A., An Introduction to Reasoning, New York, 1979.

Tsoukas H., « The Firm as a Distributed Knowledge System : a Constructionist Approach », Strategic Management Journal, 17, Special Issue, Winter 1996, p. 11-25.

Van de Ven A.H. et Poole M.S., « Explaining Development and Change in Organizations », Academy of Management Review, 20 (3), 1995, p. 510-540.

Weick K.E., Sensemaking in Organizations, Thousand Oaks, CA, Sage Publications, 1995. Westley F.R., « Middle Managers and Strategy : Microdynamics of Inclusion », Strategic Management Journal, 11 (5), 1990, p. 337-35 1. 